\title{
Diabetes and Stroke Prevention: A Review
}

\author{
Jonathan Hewitt, ${ }^{1}$ Luis Castilla Guerra, ${ }^{2}$ \\ María del Carmen Fernández-Moreno, ${ }^{3}$ and Cristina Sierra ${ }^{4}$ \\ ${ }^{1}$ Geriatric Medicine, University of Cardiff, Cardiff CF14 4XY, UK \\ ${ }^{2}$ Department of Internal Medicine, Hospital de la Merced, University of Seville, Seville, Spain \\ ${ }^{3}$ Department of Neurology, Hospital de Valme, University of Seville, Seville, Spain \\ ${ }^{4}$ Department of Internal Medicine, Hospital Clinic, University of Barcelona, Barcelona, Spain
}

Correspondence should be addressed to Jonathan Hewitt, hewittj2@cardiff.ac.uk

Received 21 May 2012; Revised 19 September 2012; Accepted 25 October 2012

Academic Editor: Graham S. Venables

Copyright ( $) 2012$ Jonathan Hewitt et al. This is an open access article distributed under the Creative Commons Attribution License, which permits unrestricted use, distribution, and reproduction in any medium, provided the original work is properly cited.

\begin{abstract}
Stroke and diabetes mellitus are two separate conditions which share multiple common threads. Both are increasing in prevalence, both are diseases which affect blood vessels, and both are associated with other vascular risk factors, such as hypertension and dyslipidemia. Abnormal glucose regulation, of which diabetes is one manifestation, is seen in up to two-thirds of people suffering from an acute stroke. Surprisingly, aggressive management of glucose after an acute stroke has not been shown to improve outcome or reduce the incidence of further strokes. More encouragingly, active management of other cardiovascular risk factors has been demonstrated to prevent stroke disease and improve outcome following a stroke in the diabetic person. Hypertension should be treated with a target of $140 / 80 \mathrm{mmHg}$, as a maximum. The drug of choice would be an ACE inhibitor, although the priority is blood pressure reduction regardless of the medication chosen. Lipids should be treated with a statin whatever the starting cholesterol. Antiplatelet treatment is also essential but there are no specific recommendations for the diabetic person. As these conditions become more prevalent it is imperative that the right treatment is offered for both primary and secondary prevention in diabetic people, in order to prevent disease and minimize disability.
\end{abstract}

\section{Introduction}

Diabetes mellitus (DM) is a common problem whose prevalence is increasing due to population aging and the growing problem of obesity. According to the World Health Organization (WHO), the prevalence of diabetes for all age groups worldwide is estimated to be approximately $2.8 \%$, and the total number of people with diabetes is projected to rise from 171 million in 2000 to 366 million in 2030 [1]. In the United States alone over 23 million people have diabetes, and the number of people with diabetes diagnosed is estimated to increase $165 \%$ between 2000 and 2050 [2].

Abnormal glucose regulation can occur in a number of different clinical situations: diabetes, impaired glucose tolerance (IGT), or in times of acute illness. Diabetes is the clinical condition present when glucose is chronically raised. It can remain undiagnosed in asymptomatic individuals while remaining a risk factor for the development of stroke.
IGT is a condition where glucose is not regulated properly but remains at levels below that of frank diabetes. Individuals are usually asymptomatic but remain at increased risk of diabetic complications and the condition often proceeds the development of diabetes. In times of medical stress blood glucose also typically rises (hyperglycaemia), in people with and without diabetes or IGT.

Diabetes is an independent risk factor for stroke disease [3]. Compared with nondiabetic patients, diabetic patients have at least twice the risk for stroke, and approximately $20 \%$ of diabetic patients will die from stroke, making it one of the leading causes of death in this population. Diabetes duration has also been shown to increase the risk of ischaemic stroke disease, with every year of diabetes duration increasing the risk by $3 \%$ [3]. Hyperglycaemia has been shown to increase the size of ischaemic stroke and worsen the clinical outcome following a stroke [4]. 
The management of diabetes and stroke disease share many characteristics, primarily due to the fact that diabetes affects blood vessels (in addition to other organ systems) and stroke is a disease of blood vessels. Further, diabetes is commonly associated with other cardiovascular risk factors such as hypertension and dyslipideamia.

Consequently aggressive management and optimisation of cardiovascular risk factors are paramount. For example, the United Kingdom Prospective Diabetes Study (UKPDS), a large exclusively diabetic cohort, showed that increased age, smoking, increased systolic blood pressure, and the presence of atrial fibrillation predicted the risk of a first stroke. With the exception of age, all are modifiable risk factors and form a routine part of the prevention of stroke disease in nondiabetic populations.

\section{Estimations of the Prevalence of Cerebrovascular Disease among People with Diabetes}

The prevalence estimates of diabetes and stroke disease vary. This is due to the method of diagnosis of diabetes, stroke disease, or the type of prevalence estimate undertaken. However, the majority of community or hospital based estimates suggest diabetes is present in about 10-25\% of people with stroke disease and stress related hyperglycaemia is found in up to two-thirds of people with an acute stroke [5]. Of which roughly half have diabetes or IGT.

For example, the Minnesota Heart Survey estimated the prevalence of diabetes in people hospitalized for stroke, $22.4 \%$ in men and $24.7 \%$ in women [6]. Barzilay and colleagues [7] recruited 5712 people all aged over 65 years, in the Cardiovascular Heart Study. They were screened for cerebrovascular disease and underwent fasting glucose measurements. In people found to have diabetes the prevalence of cerebrovascular disease was $12.6 \%$ for men and $12.7 \%$ for women. In the MRC Study of Older People, a community based questionnaire which surveyed over 15000 people aged over 75 years, the prevalence of stroke was estimated to be $15.5 \%$ in men and $12.6 \%$ in women [8].

\section{The Pathological Effects of Hyperglycaemia on the Cardiovascular (CV) System and Brain}

Multiple indirect or direct pathways that result in accelerated atherosclerosis have been proposed to explain the deleterious effects of elevated glucose levels on the cardiovascular system [9]. Indirect pathways promoted by hyperglycaemia include worsening of dyslipidaemia, especially the development of atherogenic dyslipidaemia (small dense lowdensity lipoproteins, reduced high-density lipoproteins, and increased triglyceride levels) and sympathetic nervous system dysfunction. Direct acceleration of the atherosclerotic process by hyperglycaemia is in part related to the development of endothelial dysfunction which in turn promotes vasoconstrictive, proinflammatory, and prothrombotic processes that contribute to plaque development and rupture [10].
The pathophysiology of cerebrovascular disease in patients with DM is not fully characterized, but both large and small blood vessels seem to be affected [10]. Both community and hospital based studies have not suggested any particular association between DM and any subtype of ischemic stroke [10].

\section{Prevention of Stroke Disease in Diabetic People}

4.1. Glycaemic Control. Improved glycaemic control has been shown to reduce the incidence of other common complications of diabetes, such as retinopathy, nephropathy, and neuropathy [11]. Despite this and perhaps surprisingly, better glycaemic control has not been shown to reduce the incidence of acute stroke or improve survival from acute stroke. Multiple observational and randomized controlled trials have consistently failed to demonstrate any benefit from tightly regulating blood glucose in relation to stroke disease [12-14].

Three major randomized clinical trials of intensive glucose management in persons with diabetes with a history of cardiovascular disease, stroke, or additional vascular risk factors have all failed to demonstrate a reduction in cardiovascular events, including stroke or death in the groups receiving intensive glucose therapy. These trials are the Action to Control Cardiovascular Risk in Diabetes (ACCORD) trial [15], the Action in Diabetes and Vascular Disease (ADVANCE) [16], and the Veterans Affairs Diabetes Trial (VADT) [17]. In the ACCORD trial, 10251 patients with type 2 diabetes and vascular disease or multiple risk factors were randomly assigned to an intensive treatment program targeting a glycated hemoglobin level of $<6 \%$ versus a standard program with a goal HbA1c level of $7 \%$ to $7.9 \%$. The trial was halted after a mean of 3.5 years of followup because of an increased risk of death in patients randomized to the intensive treatment program (HR, 1.22; 95\% CI, 1.01 to 1.46$)$. There was no significant difference in the rate of nonfatal stroke (HR, 1.06; 95\% CI, 0.75 to $1.50 ; P=0.72$ ) or in the primary end point, which was a composite of nonfatal heart attack, nonfatal stroke, and death due to a cardiovascular cause $(\mathrm{HR}, 0.90 ; 95 \% \mathrm{CI}, 0.78$ to $1.04 ; P=$ 0.16). The ADVANCE trial also failed to show a benefit in secondary prevention of cardiovascular events. In this trial 11140 patients with type 2 diabetes and a history of macrovascular disease or another risk factor were randomly assigned to intensive glucose control (target $<6.5 \%$ ) or standard glucose control (target HbAlc $>7 \%$ ). Thirty-two percent of subjects had a history of major macrovascular disease, including 9\% with a history of stroke. There was no significant reduction in the occurrence of macrovascular events alone (HR, $0.94 ; 95 \% \mathrm{CI}, 0.84$ to $1.06 ; P=0.32$ ) or nonfatal stroke $(3.8 \%$ in both treatment arms). In contrast to the ACCORD trial, there were no significant differences in the rate of deaths between the study groups. Finally, the VADT, consisting of 1791 veterans with type 2 diabetes assigned to intensive blood glucose treatment or standard treatment, found no significant difference between the 2 
groups in any component of the primary outcome, which consisted of time to occurrence of a major cardiovascular event, or in the rate of death due to any cause (HR, 1.07; 95\% CI, 0.81 to $1.42 ; P=0.62$ ). The results of these trials indicate that the glycemic targets should not be lowered to HbAlc $<6.5 \%$ in patients with a history of cardiovascular disease or the presence of vascular risk factors.

In the setting of acute stroke hyperglycaemia aggressive management of glucose has also failed to demonstrate benefit. The largest trial to date, the Glucose Insulin in Stroke Trial (GIST), while still underpowered, contained 933 patients [18]. They were randomized to glucose, potassium and insulin infusion, or no intervention. This trial did not show benefit in 90 mortality, which was the primary end point, or severe disability, the secondary end point.

The reasons for the lack of demonstrated benefit with tight glucose control are complex and as yet not fully explained. Hypotheses include hypoglycaemia, a significant pathology, which is far more common with tightly regulated blood glucose. Hypoglycaemia is a dangerous and unpleasant condition. Hypoglycaemia has not been directly linked to stroke disease. However, the GRASP study, which assessed the feasibility of aggressive glucose management, showed a strong association with hypoglycaemia and tight glucose control in the acute post stroke setting [19]. Further, hypoglycaemia, especially if severe and repeated, is associated with cognitive impairment. One possible explanation of the mechanism is that hypoglycaemia induces cerebrovascular damage, via direct hypoglycaemic induced neuronal damage [20].

Another suggestion for the lack of improvement is that the drugs commonly used to treat diabetes may in themselves be harmful, for example, the fluid retention and increased incidence of heart failure seen with thiazolidinedione type medications or the weight gain associated with insulin therapy.

This lack of improvement in stroke outcomes is seen across the spectrum of diabetic disease encountered in stroke disease; primary prevention, the management of acute stroke related hyperglycaemia, and secondary prevention are consistent in people with IGT and both type 1 and type 2 diabetes. While this paper would strongly recommend tight blood glucose regulation for the general improvement of nonstroke parameters in diabetic people, the evidence does not exist to suggest an improvement in stroke specific parameters with tight blood glucose control.

4.2. Hypertension. In contrast to glycaemic control, improved hypertensive management has been shown to reduce the incidence of stroke in diabetic people in a number of randomized controlled trials. The landmark trial was the HOPE study which showed in 3577 people with diabetes, aged 55 years or older, who had a previous cardiovascular event or at least one other cardiovascular risk factor and were randomly assigned ramipril or placebo those given ramipril (an angiotensin converting enzyme inhibitor (ACEI)) had a reduction in stroke risk of 33\% [21]. Another well known study, PROGRESS, compared a perindopril based treatment regime against placebo. An analysis of their diabetic participants showed that they gained the same benefit from active treatment as their nondiabetic counterparts [22].

There is also debate surrounding the optimum levels for blood pressure. Until, recently, guidelines were suggesting ever lower blood pressure targets for diabetic people, often $130 / 80 \mathrm{mmHg}$. This position has recently been challenged by analysis of the hypertensive data in the ACCORD study. Using a wide variety and combination of blood pressure medications this study demonstrated that there was no benefit in lowering systolic blood pressure below $120 \mathrm{mmHg}$ compared to $140 \mathrm{mmHg}$, in a population aged an average of 62 years [23]. It may be that future guidelines reflect this and a definitive level of acceptable blood pressure agreed, at least for the systolic component. Older people with diabetes have a greater prevalence of postural hypotension and polypharmacy; hence this level of systolic blood pressure may be especially relevant for this population.

Guidelines regarding hypertensive drug therapy vary between countries, patient characteristics, and organisations and it is beyond the scope of this paper to list them all. However, the principle should be to aggressively treat hypertension in diabetic people irrespective of the medication used to achieve the intended target range. That said, beta blockade type medications worsen glycaemia and are probably best avoided. Also in view of the fact that a large part of the evidence base is derived from the use of ACE-I, many guidelines suggest this group of medications as a first line treatment for hypertension for both primary and secondary prevention of stroke. Further, in the presence of diabetic renal disease, micro, or macroalbuminuria, or impaired glomerular filtration, inhibition of the angiotensin-renin system should be recommended as a first line treatment [24]. This can be done either using an ACE-I or through direct blockade of the angiotensin-renin II receptor (ARB); both of which have been demonstrated to preserve and improve renal function in hypertensive diabetic individuals. When using either of these drugs it is important to titrate them up to the maximum tolerated dose, because their beneficial effect will be increased and they are commonly under prescribed [25].

4.3. Cholesterol. The Heart Protection Study, amongst others, confirmed the benefit of lowering cholesterol in prevention of stroke disease. Collins and colleagues compared $40 \mathrm{mg}$ simvastatin against placebo in prevention of first stroke event in a very large, high risk population [26]. Further analysis of the diabetic participants within this cohort, including those without other cardiovascular risk factors, demonstrated the benefit of simvastatin, in preventing cardiovascular outcomes, which included stroke as part of a composite end point. Importantly, they found that even in diabetic people with initially low cholesterol $(<3 \mathrm{mmol} / \mathrm{L})$, any reduction in cholesterol was beneficial [27]. Hence, any diabetic person who has suffered stroke disease should routinely be prescribed cholesterol lowering therapy regardless of their initial cholesterol level.

The role of LDL lowering for secondary stroke prevention had been unclear until recently. To address this question the SPARCL (Stroke Prevention by Aggressive Reduction in 
Cholesterol Levels) study was undertaken [28]. In this study, 4,731 patients with prior strokes or TIAs, but no evident ischemic heart disease and no atrial fibrillation (AF), were randomized to placebo or atorvastatin $80 \mathrm{mg}$ /day. The study showed a $16 \%$ risk reduction with atorvastatin treatment for fatal or nonfatal stroke $(P=0.03)$ and a $23 \%$ reduction in risk for TIA or stroke $(P=0.001)$. There was also a reduction in coronary events (hazard ratio: $0.58 ; P<0.001$ ). A secondary analysis of the SPARCL trial, which tested the effect of treatment with atorvastatin in reducing stroke in the subgroup of subjects with type 2 diabetes mellitus or metabolic syndrome and recent stroke or transient ischemic attack, found that subjects with type 2 diabetes were at higher risk for recurrent stroke (hazard ratio $(\mathrm{HR})=1.62$; 95\% confidence interval (CI), 1.33-1.98; $P<0.001)$ and cardiovascular events and also found no difference in the effect of statin treatment in reducing these events in subjects with or without type 2 diabetes [29].

4.4. Antiplatelet Therapy. Platelets have a "key role" in atherogenesis and its thrombotic complications in subjects with diabetes. Platelets from subjects with DM, particularly from those with type 2 diabetes, exhibit increased reactivity [30]. Factors that may contribute to this are not completely elucidated and include metabolic abnormalities as hyperglycemia, hyperlipidemia, insulin resistance, and conditions as oxidative stress, inflammation, and endothelial dysfunction [31].

Besides, there is a reduced clinical efficacy ("aspirin resistance") of aspirin in diabetic compared with a nondiabetic population [32]. Hyperglycemia may be one of the mechanisms involved in this phenomenon. Increased glycation of platelets and coagulation factors may interfere with acetylation by aspirin.

For primary prevention of cardiovascular disease in diabetic people, anti-platelet agents are often started on the basis of a composite cardiovascular risk factor score. One commonly used score, the Framingham cardiovascular risk score, uses diabetes and stroke as risk factors to calculate the likelihood of future cardiovascular end points, including stroke. Recommendations about the use of aspirin as a primary preventative treatment in diabetic people does, however, remain a clinician guided decision. This is because trial data does not support the routine use of aspirin as a primary prevention, although it does appear relatively safe without an increased risk of bleeding which may have been expected [33]. Currently there is little evidence regarding other anti-platelet agents, such as clopidogrel, in relation to primary prevention of stroke disease in the diabetic person.

For the secondary prevention of stroke, the evidence base comparing aspirin and clopidogrel in the diabetic person is relatively sparse, although the CAPRIE study did compare clopidogrel and aspirin in this population. This study enrolled people with a history of cardiovascular disease, onethird of which was stroke related. The investigators subsequently looked specifically at their diabetic participants. In a post hoc analysis they suggested that clopidogrel was better than aspirin for diabetic people [34]. However, secondary prevention generally remains based on local guidelines and usually comprises of aspirin (with or without the addition of dipyridamole) or clopidogrel.

Currently, there is no evidence to suggest that a combination of aspirin and clopidogrel, even in high risk people, such as those with diabetes, has any therapeutic advantage and may actually be harmful. For example, the MATCH trial considered high risk individuals with stroke or TIA and one other risk factor. Individuals already taking clopidogrel were randomised to receive either aspirin or placebo, in addition to clopidogrel. The trial failed to show a reduction in its composite cardiovascular end point (which included stroke) but did demonstrate increased rates of bleeding when using a combination of the two drugs [35]. Further, the CHARISMA study, which compared over 15000 people with cardiovascular disease, did not show any benefit of clopidogrel and aspirin combined, when compared to aspirin alone, in their chosen end point, a composite of myocardial infarction, stroke, or cardiovascular death. Although this study did not show an increase in unwanted bleeding complications [36].

4.5. Atrial Fibrillation. Atrial fibrillation predisposes to embolic stroke disease. The risk of stroke disease varies between individuals and is often calculated using established risk stratification scores, such as the CHADS2 score, with diabetes being one factor which is often included in these scores. From these scores it is then possible to recommend the use of warfarin, which has been shown conclusively to reduce stroke [37]. Warfarin does increase bleeding risk, takes time to work, and is not suitable for all people. Hence, aspirin is often prescribed as a suitable, if less efficacious, alternative. Recently factor IIa (thrombin) inhibitors have been developed as alternatives to warfarin. Trials such as RELY [38], have demonstrated that these medications are as efficacious as warfarin. While, this trial and others like it have included diabetic people, there is as yet no specific evidence for their use in exclusively diabetic populations.

4.6. Surgical Treatments. Carotid endarterectomy for symptomatic stroke disease has been demonstrated to be the treatment of choice in the acute setting when compared to medical treatment in people with surgically significant stenosis of their carotid artery [39]. This recommendation holds true for people with diabetes and symptomatic carotid disease.

Closure of a patent foramen ovale is also recommended in certain clinical scenarios. There is relatively little evidence for the stroke population as a whole and there is no specific guidance or evidence base specific to diabetic people.

4.7. Smoking and Lifestyle Advice. Smoking and stroke disease is a very dangerous combination, either in a primary or secondary preventative setting. Those risks are multiplicative if that individual smoker also has diabetes. Advice and smoking cessation should be offered to all, regardless of diabetes.

Likewise, healthy living, good diet, exercise, moderation of alcohol, and weight loss are just as important for 
the diabetic person as those without diabetes, for stroke prevention. They should be recommended for all and form part of routine clinical care.

\section{Conclusions}

Stroke disease is common in people with diabetes. This is perhaps unsurprising given that diabetes is a major risk factor for atherosclerosis. What is more surprising is that, as yet, aggressively treating hyperglycaemia has not been shown to have beneficial outcomes in either primary or secondary prevention in stroke disease.

Aggressive management of hypertension, however, has been shown to be particularly efficacious in diabetic people. Currently, evidence would suggest target blood pressures should be $140 / 80$ as a maximum but perhaps not lower than $120 \mathrm{mmHg}$ systolic. Also an ACE-I would usually be a suitable first line agent in this population.

Cholesterol should be reduced, regardless of its starting point and other common and established primary and secondary stroke risk factors managed in diabetic people. Few, if any, management differences exist for diabetic people. What evidence has been published, specifically for the diabetic person, suggests that they too benefit from thorough stroke risk factor management.

\section{References}

[1] S. Wild, G. Roglic, A. Green, R. Sicree, and H. King, "Global prevalence of diabetes: estimates for the year 2000 and projections for 2030," Diabetes Care, vol. 27, no. 5, pp. 10471053, 2004.

[2] K. M. V. Narayan, J. P. Boyle, T. J. Thompson, S. W. Sorensen, and D. F. Williamson, "Lifetime risk for diabetes mellitus in the United States," Journal of the American Medical Association, vol. 290, no. 14, pp. 1884-1890, 2003.

[3] C. Banerjee, Y. P. Moon, and M. C. Paik, "Duration of diabetes and risk of ischeamic stroke. The Northern Manhattan Study," Stroke, vol. 43, pp. 1212-1217, 2012.

[4] V. Kothari, R. J. Stevens, A. I. Adler et al., "UKPDS 60: risk of stroke in type 2 diabetes estimated by the UK Prospective Diabetes Study risk engine," Stroke, vol. 33, no. 7, pp. 17761781, 2002.

[5] J. F. Scott, G. M. Robinson, J. M. French, J. E. O'Connell, K. G. M. M. Alberti, and C. S. Gray, "Prevalence of admission hyperglycaemia across clinical subtypes of acute stroke," The Lancet, vol. 353, no. 9150, pp. 376-377, 1999.

[6] J. M. Sprafka, B. A. Virnig, E. Shahar, and P. G. McGovern, "Trends in diabetes prevalence among stroke patients and the effect of diabetes on stroke survival. The Minnesota Heart Survey," Diabetic Medicine, vol. 11, no. 7, pp. 678-684, 1994.

[7] J. I. Barzilay, C. F. Spiekerman, L. H. Kuller et al., "Prevalence of clinical and isolated subclinical cardiovascular disease in older adults with glucose disorders: the cardiovascular health study," Diabetes Care, vol. 24, no. 7, pp. 1233-1239, 2001.

[8] J. Hewitt, L. Smeeth, C. J. Bulpitt, and A. E. Fletcher, "The prevalence of Type 2 diabetes and its associated health problems in a community-dwelling elderly population," Diabetic Medicine, vol. 26, no. 4, pp. 370-376, 2009.

[9] H. Zhang, K. C. Dellsperger, and C. Zhang, "The link between metabolic abnormalities and endothelial dysfunction in type
2 diabetes: an update," Basic Research in Cardiology, vol. 107, no. 1, article 237, 2012.

[10] L. Castilla-Guerra and M. Del Carmen Fernandez-Moreno, "Stroke in diabetic patients: is it really a macrovascular complication?" Stroke, vol. 38, no. 10, article e106, 2007.

[11] L. Blonde, "Benefits and risks for intensive glycaemic control in patients with diabetes mellitus," American Journal of the Medical Sciences, vol. 343, no. 1, pp. 17-20, 2012.

[12] F. M. Turnbull, C. Abraira, R. J. Anderson et al., "Intensive glucose control and macrovascular outcomes in type 2 diabetes," Diabetologia, vol. 52, no. 11, pp. 2288-2298, 2009.

[13] T. K. Mattila and A. De Boer, "Influence of intensive versus conventional glucose control on microvascular and macrovascular complications in type 1 and 2 diabetes mellitus," Drugs, vol. 70, no. 17, pp. 2229-2245, 2010.

[14] K. K. Ray, S. R. K. Seshasai, S. Wijesuriya et al., "Effect of intensive control of glucose on cardiovascular outcomes and death in patients with diabetes mellitus: a meta-analysis of randomised controlled trials," The Lancet, vol. 373, no. 9677, pp. 1765-1772, 2009.

[15] The Action to Control Cardiovascular Risk in Diabetes Study Group, "Effects of intensive glucose lowering in type 2 diabetes," New England Journal of Medicine, vol. 358, pp. 25452559, 2008.

[16] The ADVANCE Collaborative Group, "Intensive blood glucose control and vascular outcomes in patients with type 2 diabetes," New England Journal of Medicine, vol. 358, pp. 25602572, 2008.

[17] W. Duckworth, C. Abraira, T. Moritz et al., "Glucose control and vascular complications in veterans with type 2 diabetes," New England Journal of Medicine, vol. 360, no. 2, pp. 129-139, 2009.

[18] C. S. Gray, A. J. Hildreth, P. A. Sandercock et al., "Glucosepotassium-insulin infusions in the management of post-stroke hyperglycaemia: the UK Glucose Insulin in Stroke Trial (GISTUK)," Lancet Neurology, vol. 6, no. 5, pp. 397-406, 2007.

[19] K. C. Johnston, C. E. Hall, B. M. Kissela, T. P. Bleck, and M. R. Conaway, "Glucose regulation in acute stroke patients (grasp) trial: a randomized pilot trial," Stroke, vol. 40, no. 12, pp. 38043809, 2009.

[20] R. A. Whitmer, A. J. Karter, K. Yaffe, C. P. Quesenberry, and J. V. Selby, "Hypoglycemic episodes and risk of dementia in older patients with type 2 diabetes mellitus," Journal of the American Medical Association, vol. 301, no. 15, pp. 1565-1572, 2009.

[21] Heart Outcomes Prevention Evaluation Study Investigators, "Effects of ramipril on cardiovascular and microvascular outcomes in people with diabetes mellitus: results of the HOPE study and MICRO-HOPE substudy," The Lancet, vol. 355, no. 9200, pp. 253-259, 2000.

[22] K. Berthet, B. C. Neal, J. P. Chalmers et al., "Reductions in the risks of recurrent stroke in patients with and without diabetes: the PROGRESS Trial," Blood Pressure, vol. 13, no. 1, pp. 7-13, 2004.

[23] The ACCORD Study Group, "Effects of intensive bloodpressure control in type 2 diabetes mellitus," New England Journal of Medicine, vol. 362, pp. 1575-1585, 2010.

[24] H. H. Parving, H. Lehnert, J. Brochner-Mortensen, R. Gomis, S. Andersen, and P. Arner, "The effect of irbesartan on the development of diabetic nephropathy in patients with type 2 diabetes," New England Journal of Medicine, vol. 345, no. 12, pp. 870-878, 2001.

[25] G. F. M. Strippoli, C. Bonifati, M. Craig, S. D. Navaneethan, and J. C. Craig, "Angiotensin converting enzyme inhibitors and angiotensin II receptor antagonists for preventing the 
progression of diabetic kidney disease," Cochrane Database of Systematic Reviews, no. 4, Article ID CD006257, 2006.

[26] R. Collins, J. Armitage, S. Parish et al., "Effects of cholesterollowering with simvastatin on stroke and other major vascular events in 20536 people with cerebrovascular disease or other high-risk conditions," The Lancet, vol. 363, no. 9411, pp. 757767, 2004.

[27] R. Collins, J. Armitage, and S. Parish, "MRC/BHF Heart Protection Study of cholesterol-lowering with simvastatin in 5963 people with diabetes: a randomised placebo-controlled trial," The Lancet, vol. 361, no. 9374, pp. 2005-2016, 2003.

[28] P. Amarenco, O. Benavente, L. B. Goldstein et al., "Results of the stroke prevention by aggressive reduction in cholesterol levels (SPARCL) trial by stroke subtypes," Stroke, vol. 40, no. 4, pp. 1405-1409, 2009.

[29] A. Callahan, L. B. Goldstein, and H. Sillesen, "Risk of stroke and cardiovascular events after ischemic stroke or transient ischemic attack in patients with type 2 diabetes or metabolic syndrome: secondary analysis of the Stroke Prevention by Aggressive Reduction in Cholesterol Levels (SPARCL) trial," Archives of Neurology, vol. 68, pp. 1245-1251, 2011.

[30] A. Tufano, E. Cimino, N. M. D. . Di Minno et al., "Diabetes mellitus and cardiovascular prevention: the role and the limitations of currently available antiplatelet drugs," International Journal of Vascular Medicine, vol. 2011, Article ID 250518, 5 pages, 2011.

[31] P. Ferroni, S. Basili, A. Falco, and G. Davì, "Platelet activation in type 2 diabetes mellitus," Journal of Thrombosis and Haemostasis, vol. 2, no. 8, pp. 1282-1291, 2004.

[32] A. Y. Gasparyan, T. Watson, and G. Y. H. Lip, "The role of Aspirin in cardiovascular prevention: implications of Aspirin resistance," Journal of the American College of Cardiology, vol. 51, no. 19, pp. 1829-1843, 2008.

[33] C. Zhang, A. Sun, and P. Zhang, "Aspirin for primary prevention of cardiovascular events in patients with diabetes: a meta-analysis," Diabetes Research and Clinical Practice, vol. 87, no. 2, pp. 211-218, 2010.

[34] D. L. Bhatt, S. P. Marso, A. T. Hirsch, P. A. Ringleb, W. Hacke, and E. J. Topol, "Amplified benefit of Clopidogrel versus Aspirin in patients with diabetes mellitus," American Journal of Cardiology, vol. 90, no. 6, pp. 625-628, 2002.

[35] P. H. C. Diener, P. J. Bogousslavsky, P. L. M. Brass et al., "Aspirin and clopidogrel compared with clopidogrel alone after recent ischaemic stroke or transient ischaemic attack in high-risk patients (MATCH): randomised, double-blind, placebo-controlled trial," The Lancet, vol. 364, no. 9431, pp. 331-337, 2004.

[36] D. L. Bhatt, K. A. A. Fox, W. Hacke et al., "Clopidogrel and aspirin versus aspirin alone for the prevention of atherothrombotic events," New England Journal of Medicine, vol. 354, no. 16, pp. 1706-1717, 2006.

[37] R. G. Hart, L. A. Pearce, and M. I. Aguilar, "Meta-analysis: antithrombotic therapy to prevent stroke in patients who have nonvalvular atrial fibrillation," Annals of Internal Medicine, vol. 146, no. 12, pp. 857-867, 2007.

[38] S. J. Connolly, M. D. Ezekowitz, and S. Yusuf, "Dabigatran versus warfarin in patients with atrial fibrillation," New England Journal of Medicine, vol. 361, pp. 1139-1151, 2009.

[39] A. R. Naylor, P. M. Rothwell, and P. R. F. Bell, "Overview of the principal results and secondary analyses from the European and North American randomised trials endarterectomy for symptomatic carotid stenosis," European Journal of Vascular and Endovascular Surgery, vol. 26, no. 2, pp. 115-129, 2003. 


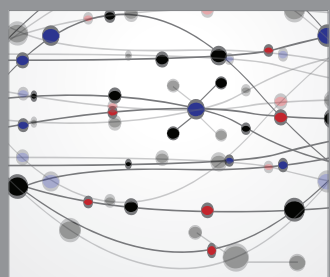

The Scientific World Journal
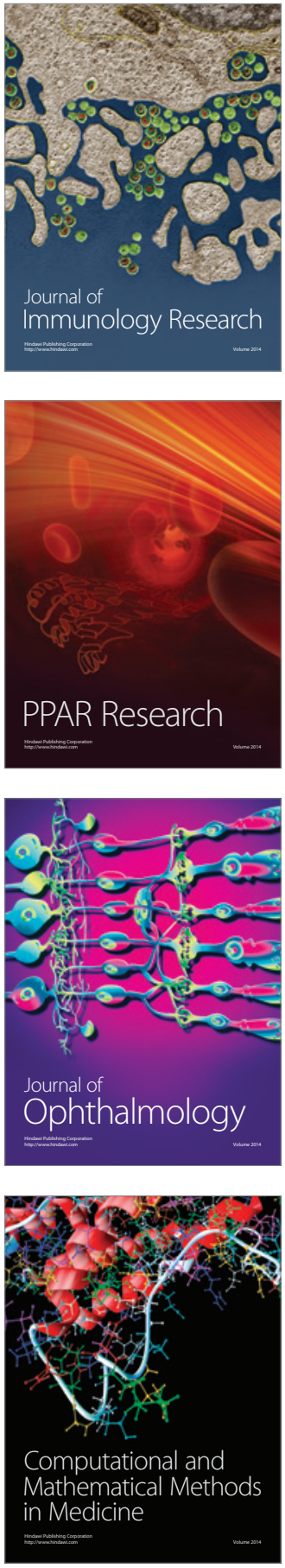

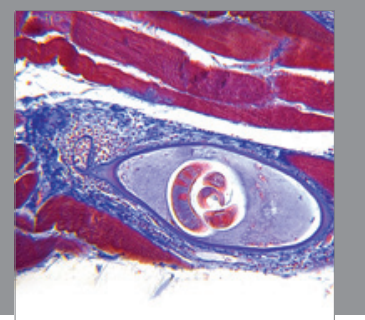

Gastroenterology

Research and Practice
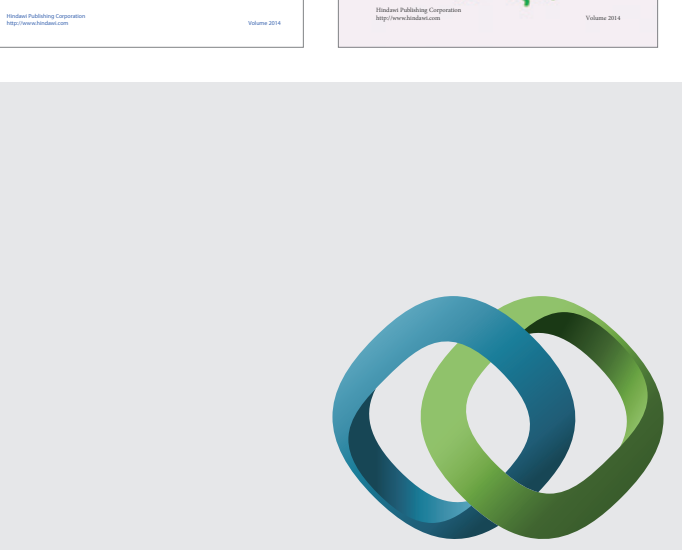

\section{Hindawi}

Submit your manuscripts at

http://www.hindawi.com
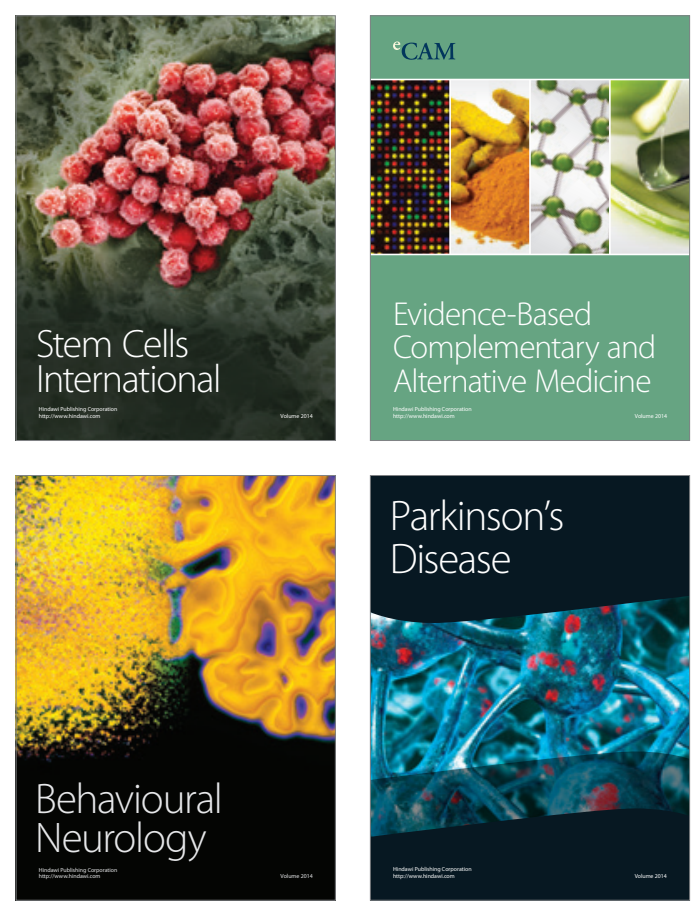

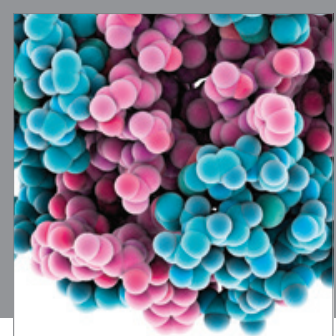

Journal of
Diabetes Research

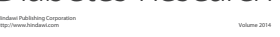

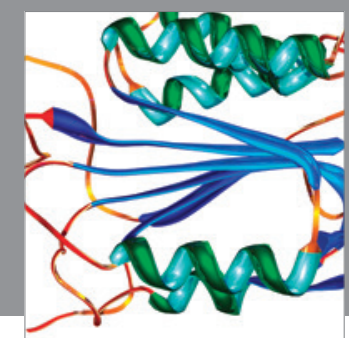

Disease Markers
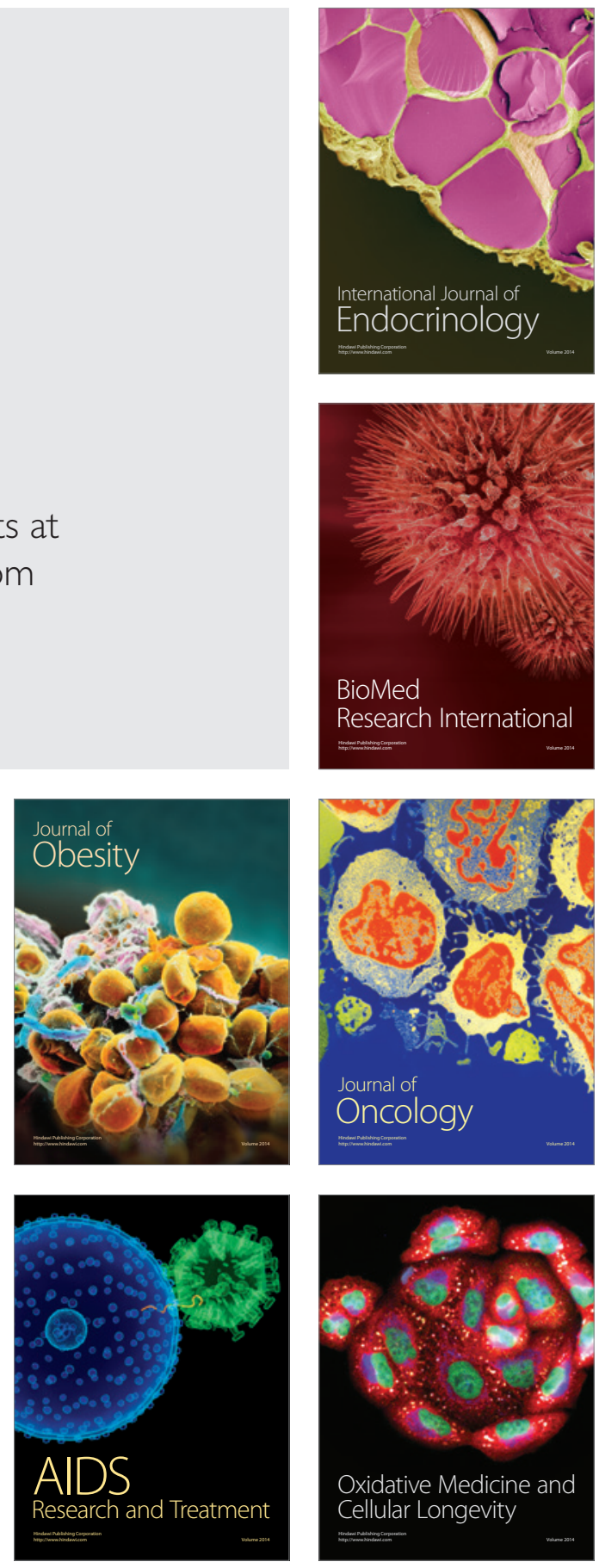